\title{
SISTEMATIZAÇÃO DA ASSISTÊNCIA DE ENFERMAGEM NO CENÁRIO HOSPITALAR: PERCEPÇÃO DOS ENFERMEIROS
}

Elisangela $\mathrm{Cogo}^{1}$, Maria Helena Gehlen², Silomar Ilha ${ }^{3}$, Claudia Zamberlan ${ }^{4}$, Hilda Maria Barbosa de Freitas ${ }^{5}$, Dirce Stein Backes ${ }^{6}$

RESUMO: Objetivou-se com este estudo conhecer o que os enfermeiros atuantes em Unidade de Terapia Intensiva de um hospital de médio porte compreendem acerca da operacionalização da Sistematização da Assistência de Enfermagem. Trata-se de uma pesquisa descritiva exploratória com abordagem qualitativa, realizada por meio de entrevista no período de março a junho de 2011. Os dados foram analisados por meio da discussão das ideias que mais se repetiram e estas foram agrupadas em elementos que as traduziram de forma sintética, resultando nas categorias Sistematização da Assistência de Enfermagem: uma necessidade efetiva no cotidiano do enfermeiro e Sistematização da Assistência de Enfermagem: um dever no cotidiano hospitalar. Pode-se dizer que os sujeitos do estudo, embora conhecedores da necessidade da Sistematização da Assistência de Enfermagem no serviço, ainda não a operacionalizaram, o que permite considerar que alguns profissionais ainda sustentam o seu saber fazer no modelo biomédico. PALAVRAS-CHAVE: Enfermagem, Operacionalização, Sistematização da assistência.

\section{SYSTEMATIZATION OF NURSING CARE IN THE HOSPITAL SCENARIO: THE NURSES' PERCEPTION}

ABSTRACT: This study aimed to investigate what the nurses working in an Intensive Care Unit in a mid-size hospital understood about the operationalization of the Systematization of Nursing Care. This research was descriptive and exploratory, and has a qualitative approach. It was undertaken in the period March - June 2011. The data was analyzed through discussion of the most-frequentlymentioned ideas. These were grouped in elements which translate them in summary form, resulting in the categories Systematization of Nursing Care: an effective necessity in the nurse's routine and Systematization of Nursing Care: a duty in the hospital routine. One may say that the research's subjects, although aware of the need for the Systematization of Nursing Care at work, did not operationalize it, which allows one to consider that some health professionals still underpin their knowledge in the biomedical model.

KEYWORDS: Nursing, Operationalization, Systematization of care.

\section{SISTEMATIZACIÓN DE LA ASISTENCIA DE ENFERMERÍA EN EL ESCENARIO HOSPITALAR: PERCEPCIÓN DE LOS ENFERMEROS}

RESUMEN: El objetivo de este estudio fue conocer lo que los enfermeros actuantes en Unidad de Terapia Intensiva de un hospital medio comprenden acerca de la operacionalización de la Sistematización de la Asistencia de enfermería. Es una investigación descriptiva exploratoria con abordaje cualitativo, realizada por medio de entrevista en el periodo de marzo a junio de 2011. Los datos fueron analizados por medio de la discusión de las ideas que más se repitieron y estas fueron agrupadas en elementos que las traducieron de forma sintética, resultando en las categorías Sistematización de la Asistencia de Enfermería: una necesidad efectiva en el cotidiano del enfermero y Sistematización de la Asistencia de Enfermería: un deber en el cotidiano hospitalar. Se puede decir que los sujetos del estudio, a pesar de conocer la necesidad de la Sistematización de la Asistencia de Enfermería en el servicio, todavía no la operacionalizaron, lo que permite considerar que algunos profesionales todavía tienen su saber hacer basado en el modelo biomédico.

PALABRAS CLAVES: Enfermería, Operacionalización, Sistematización de la asistencia.

\footnotetext{
${ }^{1}$ Enfermeira. Especializanda em Enfermagem em Saúde da Mulher pela Universidade Federal dos Pampas - UNIPAMPA.

${ }^{2}$ Enfermeira. Mestre em Educação. Professora do Centro Universitário Franciscano - UNIFRA. Membro do Grupo de Estudo e Pesquisa em Empreendedorismo Social da Enfermagem e Saúde - GEPESES UNIFRA.

${ }^{3}$ Enfermeiro. Especialista em Urgência, Emergência e Trauma. Mestrando em Enfermagem pelo Programa de Pós-Graduação em Enfermagem da Universidade Federal do Rio Grande - PPGEnf FURG. Bolsista CAPES/DS. Membro do GEPESES UNIFRA.

${ }^{4}$ Enfermeira. Doutoranda em Enfermagem pelo PPGEnf FURG. Professora do UNIFRA. Membro do Grupo Interdisciplinar de Pesquisa em Educação e Saúde - GIPES UNIFRA.

${ }^{5}$ Enfermeira, Doutoranda em Enfermagem pelo DINTER Novas Fronteiras UNIFESP/UFRJ/UFSM. Professora do UNIFRA. Membro do GEPESES UNIFRA.

${ }^{6}$ Enfermeira. Doutora em Enfermagem. Professora da UNIFRA e da FURG. Líder do GEPESES UNIFRA.
}

Autor correspondente:

Recebido: $17 / 02 / 2012$

Elisangela Cogo

Aprovado: 16/08/2012

Universidade Federal dos Pampas

Rua Pará, 516 - 97030-420 -Santa Maria-RS-Brasil

E-mail: elisangelacogo@yahoo.com.br 


\section{INTRODUÇÃO}

A organização dos serviços baseou-se durante anos em uma estrutura rígida, verticalizada, com funções pontuais e pouco desafiadoras. Neste sentido, a Enfermagem conformou-se com a cultura da ação sem reflexão acerca de novas possibilidades do ser e agir na prática assistencial e gerencial ${ }^{(1)}$.

O enfermeiro, para prestar a assistência com qualidade e humanismo, necessita inserir-se na realidade concreta de forma consciente, competente, técnica e científica. Desta forma, a implantação da Sistematização da Assistência de Enfermagem (SAE), que caracteriza-se pela organização e pelo planejamento das ações no cuidado, de maneira contínua e processual, o que vem demonstrando melhoria no bem estar dos clientes hospitalizados e qualificando também os saberes da equipe de enfermagem ${ }^{(2)}$.

Esta sistematização do serviço proporciona organização no desenvolvimento do cuidado de enfermagem, desde sua base teórico-filosófico, por ser um instrumento do processo de trabalho que deve ser incorporado do ensino ao serviço de Enfermagem, através do planejamento, da organização e da execução do cuidado e do próprio gerenciamento da assistência de Enfermagem ${ }^{(1)}$.

Todavia, observa-se que as ações do enfermeiro são desenvolvidas, muitas vezes, desvinculadas da SAE, o que dicotomiza a singularidade do indivíduo para o atendimento de suas particularidades. Por outro lado, ao se problematizar essa realidade, percebe-se a necessidade do enfermeiro transformar essa ordem linear e empreender a operacionalização da SAE.

Neste contexto, a Enfermagem conformou-se, basicamente, com uma cultura do "fazer" sem, contudo, refletir acerca de novas possibilidades do ser e agir na prática assistencial e gerencial. Porém, as preocupações com os processos de melhoria do cuidado em consonância com as necessidades dos clientes aliam a tecnologia à transformação social, culminando com a flexibilidade, inovação, criatividade, qualidade dos serviços, humanização da assistência entre outros, para garantir o espaço profissional com responsabilidade social ${ }^{(2)}$.

A SAE torna-se uma estratégia metodológica que promove a liderança do ser enfermeiro, transformando o cuidado no cenário hospitalar através da ética, compromisso e responsabilidade. A competência da liderança do enfermeiro é fundamental nesse processo, pois o sucesso das organizações relaciona-se com a capacidade do líder em influenciar as pessoas a atuarem de modo ético-profissional, o que exige a construção de laços de confiança na equipe de enfermagem, a fim de possibilitar o trabalho de maneira coletiva, no intuito de alcançar a implementação da $\mathrm{SAE}^{(3)}$. Entretanto, não basta somente operacionalizar, é necessário criar processos de educação permanente com a equipe de enfermagem, visando um cuidado humanizado, circular e integrador ${ }^{(2-3)}$.

Considera-se que a SAE é uma necessidade no serviço. Desse modo, a informação de saúde deverá vincular-se a tecnologia garantindo a sua universalização de forma rápida e eficaz, promovendo a socialização nas diferentes áreas. Com isso, a gestão e organização desse recurso devem definir com clareza o tipo de informação que deverá ser documentada e partilhada de forma a consolidar um Sistema de Informação de Enfermagem (SIE) adequado e baseado nos princípios dessa sistematização ${ }^{(4)}$.

$\mathrm{Na}$ informatização da SAE é imprescindível à utilização de um sistema padronizado de linguagem do processo assistencial, para que se possa realizar a captura dos dados desse processo, agrupá-los, classificá-los, analisá-los e transformá-los em informações. A padronização pressupõe a utilização de um conjunto de termos pré-estabelecidos que expressem situações do processo saúde/doença que podem ser modificadas por intervenções e ações de enfermagem.

Acredita-se que a SAE estimula o saber fazer do enfermeiro de maneira a atender as necessidades do paciente, sendo uma estratégia que facilita e possibilita a construção da autonomia profissional. Frente a essas considerações emergiu a questão norteadora do estudo: como se desenvolve a operacionalização da SAE pelos enfermeiros no cenário hospitalar?

Nesse delineamento, objetivou-se conhecer o que os enfermeiros compreendem acerca da operacionalização da Sistematização da Assistência de Enfermagem.

\section{MÉTODO}

O presente estudo aborda uma pesquisa exploratória descritiva de caráter qualitativo. $\mathrm{O}$ caráter exploratório visa proporcionar maior familiaridade com o problema, com vistas a desenvolver, esclarecer, modificar conceitos e ideias para estudos posteriores, torná-lo mais explícito. Já o caráter descritivo tem como objetivo primordial a descrição das características de determinadas populações ou fenômenos; por meio da precisão dos detalhes ${ }^{(5)}$. 
A coleta de dados foi realizada no período de março a junho de 2011, com enfermeiros de Unidade de Tratamento Intensivo de hospital de médio porte na região central do Rio Grande do Sul. Assim, foram incluídos apenas os enfermeiros atuantes nos turnos da manhã, tarde e noite e excluídos os que estavam de atestado ou em férias. Atenderam aos critérios de inclusão e aceitaram participar de maneira voluntária, após assinatura do Termo de Consentimento Livre e Esclarecido, oito enfermeiros.

O instrumento de coleta de dados foi constituído de entrevista semiestruturada, contemplando as seguintes questões norteadoras: Enquanto profissional enfermeiro já teve contato com a SAE no seu ambiente de trabalho? Como você enfermeiro reconhece o ser humano? Você profissional é a favor da implantação da SAE no setor em que trabalha? Por quê? Você enquanto enfermeiro acredita que a SAE seja importante e possa melhorar a qualidade do cuidado prestado na unidade em que trabalha? Por quê?

A análise dos dados ocorreu por meio da categorização das respostas, ou seja, foram discutidas as idéias que mais se repetiram e estas foram agrupadas em elementos que as traduziram de forma sintética. Tratou-se de uma classificação e elementos constitutivos de um conjunto, que por diferenciação, seguidamente, inferiu o agrupamento ${ }^{(6)}$.

As questões éticas foram relevantes para desenvolver esse estudo, visando não correr o risco de invalidar a pesquisa. Assim solicitou-se o consentimento dos indivíduos, respeitada a resolução do CNS 196/96, assegurando o sigilo e o anonimato dos sujeitos7. Os participantes foram identificados por nome de flores tomando-se cuidado para a não manipulação dos dados. Esse estudo foi aprovado pelo comitê de ética do Centro Universitário Franciscano - UNIFRA e registrado no CONEP sob n. 391.2010.2.

\section{RESULTADOS}

Ao conhecer a operacionalização a assistência de enfermagem, percebeu-se a necessidade de sua implementação como instrumento de trabalho e valorização do saber e fazer do enfermeiro. Da análise dos dados emergiram as categorias: Sistematização da Assistência de Enfermagem: uma necessidade efetiva no cotidiano hospitalar e Sistematização da Assistência de Enfermagem: um dever no cotidiano hospitalar, descritas a seguir.

\section{Sistematização da Assistência de Enfermagem: uma necessidade efetiva no cotidiano do Enfermeiro}

Na realidade investigada, é imprescindível que o enfermeiro operacionalize a SAE efetivando-a como uma necessidade emergente do serviço de enfermagem que irá oportunizar o desenvolvimento da autonomia e a competência da liderança.

Operacionalizar a SAE é uma questão organizacional, tanto bio-tecnicista quanto humana. (Lírio)

[...] seria ótimo, porém a equipe talvez não respeite esta nova etapa e nem o médico, pois quando cheguei prescrevi alguns cuidados como os que fazíamos em outra instituição e ele não gostou desta interferência. (Rosa)

Fica evidente que embora os respondentes reconheçam a necessidade de uma SAE, na realidade, o processo de implantação, ainda se constitui um grande desafio principalmente para lideranças dispostas a desencadear o processo. Para os enfermeiros, a operacionalização da SAE inicia-se no desenvolvimento profissional, ainda na graduação e se estende ao labor, como referem às falas:

Meu contato com a SAE ocorreu na faculdade, muito breve [...] deveria ter sido mais valorizada e estimulada. (Cravo)

Meu contato inicial foi durante a formação posteriormente aprofundei meus conhecimentos por achar importante a valorização de uma linguagem padronizada, e onde eu trabalhei, utilizávamos a SAE. (Jasmim)

Os assuntos relativos a esta temática constituem um objeto de preocupação dos enfermeiros em diferentes âmbitos de atuação, sejam no ensino ou na assistência de enfermagem, devido ao distanciamento da temática do vivido no cotidiano. Para os sujeitos da pesquisa, há o reconhecimento da SAE para o atendimento das necessidades do cliente como ser único, de acordo com as evidências em seus discursos descritos:

O ser humano é único e necessita de cuidados individualizados, respeitando suas necessidades. (Cravo)

[...] como um ser único e multidimensional, bio-psicosócio-espiritual. (Lírio) 
Ao conhecer a operacionalização à assistência de enfermagem, observou-se a necessidade de sua operacionalização como instrumento de trabalho e exercício da competência de liderar do enfermeiro, atendimento das particularidades do paciente hospitalizado, organização e visibilidade do cuidado de enfermagem.

\section{Sistematização da Assistência de Enfermagem: um dever no cotidiano hospitalar}

A SAE, embora necessária, é ainda compreendida como apenas uma possibilidade que poderá organizar o fazer profissional, pois não é uma realidade efetivada no cotidiano, já que todos os participantes do estudo não visualizam a sua operacionalização, como referem as seguintes falas:

Com a SAE acredito que seria melhor o andamento da unidade e dos pacientes. (Cravo)

A SAE nos guiará mais objetivamente frente ao cuidado com o paciente [...]. Todavia falta a implantação do sistema. (Jasmim)

\section{A SAE nos trará organização e qualidade aos cuidados prestados com o paciente. (Lírio)}

[...] seria uma conquista e valorização da enfermagem. (Rosa)

As percepções dos enfermeiros, apontadas nas falas, revelam que consideram o processo de construção da SAE, de fundamental importância para o momento atual, no que diz respeito à organização e uma melhor qualidade aos cuidados prestados aos pacientes. Evidencia-se também que reconhecem a importância no que diz respeito à valorização do ser enfermeiro, como evidencia-se nas falas dos profissionais:

Deixaremos de depender exclusivamente do médico para prescrever cuidados básicos, nos fornecerá mais autonomia. (Cravo)

A SAE nos guiará de maneira mais objetiva frente ao cuidado com o paciente, demonstrará onde estão as falhas e onde deveremos melhorar frente ao cuidado, nos dará mais autonomia. (Rosa)

A SAE será importante na organização e na qualidade dos cuidados prestados [...] mostrará os pontos que de- vem ser melhorados e como resolver esses problemas. (Jasmim)

Os enfermeiros, além de considerarem importante para organização do cuidado, relacionam a operacionalização desse sistema, com a visibilidade do profissional, pois como dito nas falas, a SAE torna visível o trabalho da enfermagem. A operacionalização da SAE deve transcender da possibilidade para a realidade investigada, pois a mesma é um dever legal que atende às necessidades dos pacientes e valoriza a qualificação do corpo de conhecimentos organizacionais do cuidado de enfermagem.

\section{DISCUSSÃO}

O exercício profissional é determinante do espaço social das profissões, as quais se inserem na multidimensionalidade desse espaço social que é complexo, por vezes, exigente. O enfermeiro para prestar a assistência de enfermagem humanizada com qualidade, necessita inserir-se na rede social de cuidados de forma consciente, competente, tanto técnica quanto cientificamente. Desta forma, o processo de enfermagem hospitalar emerge com o objetivo de organizar o serviço, ou seja, garante a autonomia profissional através de uma sistematização das ações de enfermagem ${ }^{(2)}$.

Todavia, na realidade investigada, a implementação dessa sistematização ainda não se constitui como uma realidade, mesmo que ocorra empenho por parte dos profissionais enfermeiros. Esse fato faz com que a SAE seja, até os dias atuais, um desafio a ser enfrentado pelos profissionais enfermeiros dessa instituição.

A implementação da SAE mostra-se como uma estratégia para o exercício da liderança no ensino ao serviço que instiga e compromete a equipe de enfermagem para esta nova realidade ${ }^{(8)}$. A SAE não é um fenômeno natural e sim resultante da liderança do enfermeiro que pode ser considerada também como uma coletânea de instrumental tecnológico desenvolvido e adquirido ao longo da formação profissional e aperfeiçoado em atividades de educação permanente, que resultam em uma prática reflexiva e crítica ${ }^{(9)}$.

Como visto nos dados desta pesquisa, os enfermeiros relataram que não obtiveram embasamento suficiente a respeito da operacionalização da SAE durante o processo de formação. Talvez esse seja um fator prejudicial para os processos organizacionais, bem como para tomadas de decisões importantes, pois, enquanto processo organizacional, a SAE oferece subsídios para o desenvolvimento 
de métodos interdisciplinares e humanizados de cuidado. Pode ser considerada um indicativo da autonomia profissional e garantia da organização do serviço de enfermagem hospitalar. No entanto, percebe-se ainda, o cuidado de enfermagem fortemente centrado na doença e não no ser humano, enquanto sujeito ativo e participativo do processo de cuidar ${ }^{(2)}$.

A esse respeito, os enfermeiros relatam que a SAE é mais do que uma vontade e sim uma necessidade efetiva no cotidiano do enfermeiro, pois virá a atender as necessidades dos indivíduos, e valorizar o corpo de conhecimento organizacionais do cuidado de enfermagem. Entretanto, no ambiente onde desenvolveu-se o estudo, pode-se perceber que embora os enfermeiros reconheçam a importância da SAE, estes visualizam ainda como apenas uma futura possibilidade que poderá vir a organizar o cotidiano de trabalho dos profissionais que no setor atuam, deixando visível que essa operacionalização, embora idealizada não está efetivada e muito menos concretizada.

A implementação da SAE atende às necessidades do paciente, considerando, constantemente, a evolução clínica e os cuidados particulares constantes no cotidiano da hospitalização. A sistematização requer do profissional interesse em conhecer o paciente como indivíduo, utilizando, para isso, seus conhecimentos teóricos científico, além de orientação e qualificação da equipe de enfermagem para as ações sistematizadas ${ }^{(10)}$. Assim, evidencia-se a SAE como possível determinante para o desenvolvimento de processos mais interativos e multidimensionais e, ao mesmo tempo, a necessidade de articular formação profissional com práticas profissionais na saúde e os órgãos de classe ${ }^{(2)}$.

Neste aspecto, denota-se que a operacionalização da SAE é uma possibilidade que requer interesse e comprometimento, já que se constitui como orientação para o exercício da liderança do enfermeiro. Essa atividade é privativa do enfermeiro, e se implementada, o profissional, utilizará esta de método e estratégia de trabalho científico para a identificação das situações de saúde/doença, subsidiando ações de assistência de Enfermagem que possam contribuir para promoção, prevenção, recuperação e reabilitação da saúde do indivíduo, da família e da comunidade ${ }^{(11)}$.

Os enfermeiros, manifestam serem conhecedores da importância desta operacionalização para melhoria de sua prática profissional, bem como instrumento para o exercício da competência de liderar. Assim, a implantação da SAE, a partir de conhecimento específico e de reflexão crítica e problematizadora acerca da organização do trabalho de enfermagem, constitui-se na percepção dos enfermeiros investigados, instrumento de fundamental importância para que o enfermeiro possa gerenciar e otimizar a assistência de enfermagem de forma organizada, segura, dinâmica e competente ${ }^{(2)}$.

Neste processo, o enfermeiro, poderá atuar de maneira significativa, tendo em vista, seu trabalho ser baseado na identificação de respostas humanas e no estabelecimento de estratégias que proporcionem a recuperação da saúde ou a melhoria do bem-estar individual ou coletivo12. Assim, há necessidade de desenvolver métodos que permitam estabelecer relações dialógicas e influências recíprocas entre as partes e o todo.

É preciso desenvolver mecanismos inovadores, capazes de focalizar as relações multiprofissionais com os diferentes meios dos sistemas abertos que estão em constante movimento frente suas influências múltiplas e imprevisíveis ${ }^{(2)}$. Enquanto a valorização do papel do enfermeiro, a SAE mostra-se, para além da sua face burocratizada e mistificada na prática cuidativa de enfermagem ${ }^{(13)}$. Caracteriza-se, segundo os enfermeiros deste estudo, como um dever no cotidiano hospitalar. Além de proporcionar maior qualidade à assistência, propicia, também, na concepção dos enfermeiros, maior eficiência, autonomia e cientificidade à profissão, garantindo maior valorização e reconhecimento enquanto um espaço de novas conquistas e uma mudança cultural no papel do enfermeiro.

Os Enfermeiros contribuem ainda que a operacionalização da SAE vem a contemplar mais as particularidades dos sujeitos hospitalizados pela organização da assistência de enfermagem, assim como uma visibilidade profissional aos enfermeiros. Além disso, potencializará um respaldo mais seguro através do registro, que garante a continuidade/complementaridade multiprofissional, promovendo assim, aproximação enfermeiro-usuário. Ainda evidencia-se a importância da SAE enquanto processo dinâmico capaz de dar conta da continuidade do trabalho entre as equipes, estreitando laços profissionais entre a equipe multiprofissional ${ }^{(2)}$.

\section{CONCLUSÕES}

A SAE é uma necessidade que deve se estender do desenvolvimento profissional para o cotidiano do serviço da enfermagem por meio da sua operacionalização que permite a organização, o planejamento de ações sistematizadas de gerenciamento e o cuidado do cliente que está no ambiente hospitalar. A mesma atende as necessidades do paciente em sua complexida- 
de, constroi a autonomia profissional, ao desconstruir o fazer e o repetir pautados nas prescrições curativas que sustentam o modelo biomédico no atendimento dos agravos e no cuidado à saúde.

Os sujeitos do estudo, embora conhecedores da necessidade da SAE na UTI, ainda não a operacionalizaram, o que nos permite considerar que alguns profissionais ainda sustentam o seu saber fazer no modelo biomédico, que prevalece ainda nos dias atuais sendo utilizado por muitas instituições. Cabe enfatizar que as tendências de informatizar a SAE agregam tecnologia e saberes, bem como a qualificação do saber fazer do enfermeiro, o que se desvela em um desafio para realidade investigada.

Como contribuição deste estudo, em conjunto com a equipe de informática da referida instituição, em breve será desenvolvido o sistema de informação para coleta de dados e desenvolvimento da SAE, que proporcionará aos enfermeiros o registro informatizado de forma individualizada, eficiente e rápida, bem como facilitará a programação do software para a SAE em todo o hospital.

Fica então o compromisso acadêmico de continuidade da investigação com estudos que possam contribuir para que a necessidade da SAE se operacionalize, ao transcender a possibilidade para a sua efetividade.

\section{REFERÊNCIAS}

1. Backes DS, Schwartz E. Implementação da Sistematização da Assistência de Enfermagem: desafios e conquistas do ponto de vista gerencial. Rev. Ciênc. cuid. Saúde 2005;4(2):182-8.

2. Nascimento KC, Backes DS, Koerich MS, Erdmann AL. Sistematização da Assistência de Enfermagem: vislumbrando um cuidado interativo, complementar e multiprofissional. Rev. Esc. Enferm. USP. 2008;42(4):643-8.

3. Morin E. A cabeça bem feita: repensar a reforma, reformar o pensamento. $19^{\mathrm{a}} \mathrm{ed}$. Rio de Janeiro: Bertrand Brasil, 2011.

4. Chaves LD. Sistematização da assistência de enfermagem: considerações teóricas e aplicabilidade. São Paulo: Martinari, 2009.

5. Minayo MCS. O desafio do conhecimento: pesquisa qualitativa em saúde. $11^{\mathrm{a}}$ ed. São Paulo: Hucitec Abrasco, 2008.

6. Bardin L. Análise de conteúdo. $4^{\mathrm{a}}$ ed. Lisboa: Edições; 2009.
7. Ministério da Saúde (BR). Conselho Nacional de Saúde. Diretrizes e normas regulamentadoras de pesquisa envolvendo seres humanos. Resolução n. 196, de 10 de outubro de 1996. Brasília; 1996.

8. Souza R, Shimizu HE. Análise da implementação da Sistematização da Assistência de Enfermagem em uma unidade de reabilitação. Rev. bras. enferm. 2010;63(2):222-9.

9. Alfaro-Lefevre R. Aplicação do processo de enfermagem: promoção de cuidado colaborativo. $5^{\text {a }}$ ed. Porto Alegre: Artmed; 2005.

10. Malucelli A, Otemaier KR, Bonnet M, Cubas MR, Garcia TR. Sistema de informação para apoio à Sistematização da Assistência de Enfermagem. Rev. bras. enferm. 2010;63(4):629-36.

11. Conselho Federal de Enfermagem. Resolução COFEN358/2009. Dispõe sobre a Sistematização da Assistência de Enfermagem e a implementação do Processo de Enfermagem em ambientes públicos ou privados, em que ocorre o cuidado profissional de Enfermagem e dá outras providências. [Internet]. Brasília (DF); 2009 [acesso em 07 abr 2010]. Disponível: http://site. portalcofen.gov.br/node/4384

12. Nascimento LKAS, Medeiros ATN, Saldanha EA, Tourinho FSV, Santos VEP, Lira ALBC. Sistematização da assistência de enfermagem a pacientes oncológicos: uma revisão integrativa da literatura. Rev. gauch. enferm. 2012;33(1):177-85.

13. Silva LWS, Nunes ECDA, Souza DM, Santos CS, Pereira LC. Sistematização da Assistência de Enfermagem - a práxis no Ser-saber-fazer o cuidado. Cogitare enferm. 2011; 16(3):560-4. 